\title{
Dynamic Characteristics Analysis of a Seismic Vibrator-Ground Coupling System
}

\author{
Liu Jun, Huang Zhi-Qiang, Li Gang, Li Shi-De, Liu Xian, and Zhang Kai \\ School of Mechatronic Engineering, Southwest Petroleum University, No. 8 Xindu Avenue, Xindu District, \\ Chengdu, Sichuan 610500, China
}

Correspondence should be addressed to Liu Jun; 99803392@qq.com

Received 13 March 2017; Accepted 22 May 2017; Published 4 July 2017

Academic Editor: Ivo Caliò

Copyright (C) 2017 Liu Jun et al. This is an open access article distributed under the Creative Commons Attribution License, which permits unrestricted use, distribution, and reproduction in any medium, provided the original work is properly cited.

\begin{abstract}
A mathematical model to describe the seismic vibrator and ground coupling is proposed based on dynamic analyses of the vibrator baseplate structure and properties of soil. This mathematical model is solved using Newmark's method. It produces reasonable results and the trend of amplitude envelope follows the experimental data. With this model, characterization of the vibrator-ground coupling is performed. Modal analysis study shows that the motions between the vibrator reaction mass and baseplate experience a combination of a first-order vibration mode and a second-order vibration mode. The corresponding frequencies of two vibration modes are located at $2.77 \mathrm{~Hz}$ and $488.7 \mathrm{~Hz}$, respectively. As the frequency goes up, the motion behavior of the reaction mass and baseplate is dominated by the second-order vibration mode. It is realized that the second-order vibration mode is responsible for the phase difference between the input sweep signal and the output force signal. Furthermore, the impact of the coupling system parameters on the vibrator output force is also investigated. It is observed that the vibrator output force decreases as the sweep frequency increases. The weight ratio of the reaction mass and the baseplate has an impact on the vibrator output force.
\end{abstract}

\section{Introduction}

Due to efficient operation and being environment friendly, seismic vibrators (Figure 1) have become a main energy source on land in oil and gas exploration. With extension of seismic acquisition frequency bandwidth towards low and high ends, seismic vibrators face more challenges to produce a predictable and invariant output force signal. To achieve this goal, it is very necessary to understand the impact of key parameters in the vibrator-ground system on the vibrator output force [1].

Chapman et al. [2] presented a new design of the vibrator actuator to improve the vibrator output force in the frequency range of $200 \mathrm{~Hz}$. They stressed that the weight and stiffness of the vibrator baseplate played a big role in extension of the vibrator output force bandwidth towards $200 \mathrm{~Hz}$. Sallas and Weber [3] used a mass-spring-damper model to describe the vibrator system and the coupled ground system. Under rigid-body assumptions of reaction mass and baseplate, they theoretically demonstrated that a vibrator output force could be estimated using a weighted-sum method that is summing reaction mass and baseplate acceleration weighted by their respective masses. This weighted-sum ground force estimate provided a mean to approach the vibrator-ground force. However, this weighted-sum ground force estimate was proved to be valid in a narrow frequency bandwidth by many researchers [4-8].

Wei $[9,10]$ demonstrated that the frequency bandwidth of the vibrator output force was constrained by hydraulic and mechanical limits of the vibrator system. Based on experimental testing results, he derived formulas that can be used to estimate the maximum fundamental force from the vibrator at low frequencies. Subsequently, Wei [11] simulated the dynamic behavior of the vibrator baseplate as well as its coupled ground using finite element analysis tools. Then, he [11] concluded that the vibrator performance was significantly affected by the baseplate stiffness.

To improve the vibrator force output towards a wide frequency range, one way is to design broadband sweeps using nonlinear sweep design techniques $[12,13]$. The other way is to improve the performance of fundamental components in the vibrator mechanical and hydraulic system [14, 15]. 


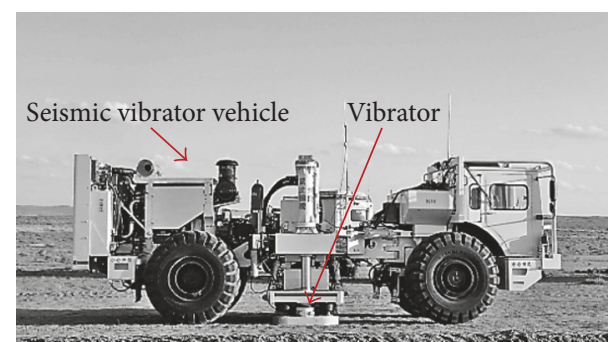

FIGURE 1: Seismic vibration system and vibrator.

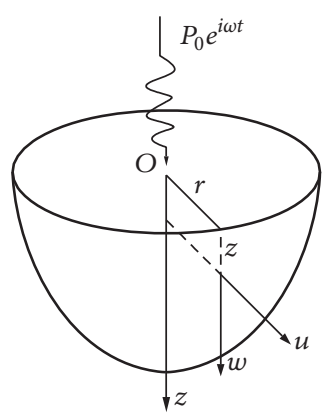

FIGURE 2: Half-infinite space under a concentrated vertical harmonic disturbance force.

Although improvements of individual components in the vibrator system are seen [5-16], the theoretical progress on the seismic vibrator design moves very slowly. This is because the dynamics between the vibrator and ground coupling are unclear.

The purpose of this paper is to present a mathematical model that is used to simulate the vibrator-ground coupling. Next, an impact of vibrator system parameters on the vibrator-ground coupling is investigated. Last, an impact of these parameters on the quality of the vibrator force output is performed.

\section{Ground Stiffness and Damping}

2.1. Surface Displacement Field of a Half-Infinite Ground Space under Concentrated Vertical Harmonic Disturbances. The displacement field of a half-infinite ground space under a concentrated vertical harmonic disturbance force $P_{0} e^{i \omega t}$ (Figure 2) can be written as follows [17, 18]:

$$
\begin{aligned}
& \rho \frac{\partial^{2} u}{\partial t^{2}}=(\lambda+2 \mu) \frac{\partial \bar{\varepsilon}}{\partial r}+2 G \frac{\partial \bar{\omega}_{\theta}}{\partial z}, \\
& \rho \frac{\partial^{2} w}{\partial t^{2}}=(\lambda+2 \mu) \frac{\partial \bar{\varepsilon}}{\partial z}+2 G \frac{1}{r} \frac{\partial}{\partial r}\left(r \bar{\omega}_{\theta}\right),
\end{aligned}
$$

with boundary conditions

$$
\begin{aligned}
\sigma_{z}(r, 0, t) & =\sigma(r) e^{i \omega t}, \\
\tau_{r z}(r, 0, t) & =0, \\
\lim _{r \rightarrow 0} \sigma(r) & =p_{0},
\end{aligned}
$$

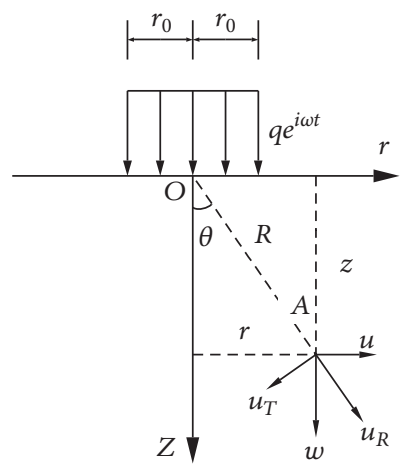

FIGURE 3: Half-infinite space model for circular baseplate vibration.

where $G, \lambda$, volume strain $\bar{\varepsilon}$, and rotational component $\bar{\omega}_{\theta}$ refer to Table 3 and, here, $\rho, E$, and $\mu$ are the density, elastic modulus, and Poisson's ratio of the ground, respectively. $u$ and $w$ are displacement components and $\sigma$ and $\tau$ are stress components.

The surface displacement field of the half-infinite space can be derived as follows:

$$
\begin{aligned}
& u(r, 0, t)=\frac{p_{0} e^{i \omega t}}{2 \pi G} \int_{0}^{\infty} \frac{p^{2}\left(2 p^{2}-k^{2}-2 \alpha \beta\right)}{\phi(p)} J_{1}(p r) d p, \\
& w(r, 0, t)=\frac{p_{0} e^{i \omega t}}{2 \pi G} \int_{0}^{\infty} \frac{k^{2} p a}{\phi(p)} J_{0}(p r) d p,
\end{aligned}
$$

where $J_{0}(p r)$ and $J_{1}(p r)$ are, respectively, zeroth-order and first-order Bessel functions of the first kind and $\phi(p), \alpha, \beta, h$, $k, v_{s}$, and $v_{p}$ refer to Table 3 .

The second formula in (3) yields the surface displacement component of the half-infinite space under a vertical disturbance as

$$
w(r, 0, t)=-\frac{p_{0} a_{0}}{G r} e^{i \omega t}\left(f_{1}+i f_{2}\right),
$$

where $a_{0}=\omega r \sqrt{\rho / G}$ is the dimensionless frequency and $f_{1}$ and $f_{2}$ are the functions of $a_{0}$. The specific expression refers to Table 3 .

2.2. Wave Displacement Field of a Circular Baseplate under a Simple Harmonic Load. Using the wave model for a dynamic half space presented in Section 2.1, the displacement field of an infinite half space with a circular surface under a simple harmonic load can be derived as shown in Figure 3.

A vibrational coupling model of a circular baseplate and the ground is shown in Figure 3, where $u, w, u_{r}$, and $u_{T}$ denote, respectively, the displacement components along the horizontal axis $r$, the vertical axis $Z$, and the direction $\overrightarrow{O A}$. 


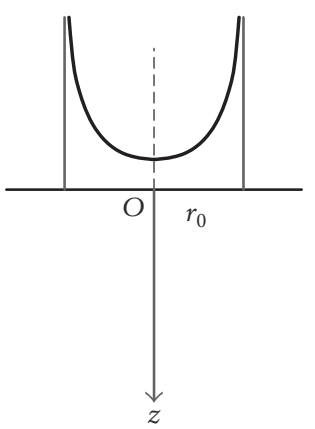

(a)

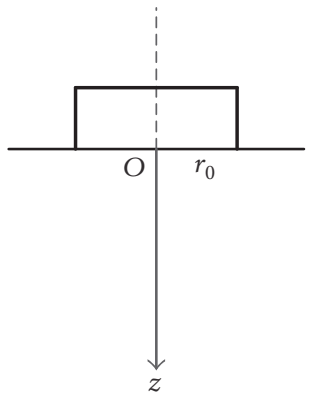

(b)

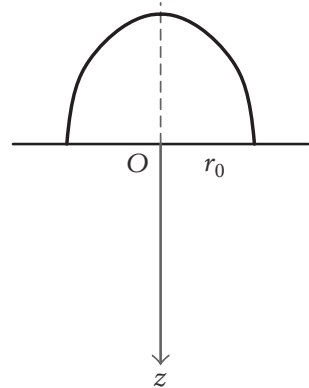

(c)

Figure 4: Distribution of reaction force imposed on the bottom of the baseplate.

The displacements produced by a body wave are given as follows $[19,20]$ :

$$
\begin{aligned}
& u(r, \mathrm{z}, t) \\
& =\frac{i q r_{0}^{2} \cos \theta}{2 G R}\left\{-\frac{1}{v^{3}} \frac{\sin 2 \theta \sqrt{\left(1 / v^{2}\right) \sin ^{2} \theta-1}}{F_{0}(\sin \theta / v)} e^{-i k R}\right. \\
& \left.+\frac{i \sin \theta\left(1 / v^{2}-2 \sin ^{2} \theta\right)}{F_{0} \sin \theta} e^{-i h R}\right\} e^{i \omega t}, \\
& w(r, \mathrm{z}, t) \\
& =\frac{i q r_{0}^{2} \cos \theta}{2 G R}\left\{\frac{1}{v^{3}} \frac{2 \sin ^{2} \theta \sqrt{\left(1 / v^{2}\right) \sin ^{2} \theta-1}}{F_{0}(\sin \theta / v)} e^{-i k R}\right. \\
& \left.+\frac{i \cos \theta\left(1 / v^{2}-2 \sin ^{2} \theta\right)}{F_{0} \sin \theta} e^{-i h R}\right\} e^{i \omega t}, \\
& u_{R}=\frac{q r_{0}^{2}}{2 G R} \frac{\cos \theta\left(1 / v^{2}-2 \sin ^{2} \theta\right)}{F_{0} \sin \theta} e^{-i h R} e^{i \omega t}, \\
& u_{T}=\frac{i q r_{0}^{2}}{2 G R v^{3}} \frac{\sin 2 \theta \sqrt{\left(1 / v^{2}\right) \sin ^{2} \theta-1}}{F_{0}(\sin \theta / v)} e^{-i k R} e^{i \omega t},
\end{aligned}
$$

where $F_{0}(x)$ and $v$ refer to Table 3 .

The stress field of the circular baseplate-ground system under the action of a harmonic disturbance can be obtained from (5). To determine the vertical displacement component of the circular baseplate $w(r, 0, t)$ with $\theta=0$, the distribution of the reaction force imposed on the bottom of the baseplate needs to be determined.

The force beneath the baseplate is determined by the stress distribution pattern beneath the baseplate. Three stress distribution patterns, which are (a) a static stiffness, (b) a uniform distribution, and (c) a parabolic distribution, are assumed (Figure 4 ). The stress boundary $\sigma_{z}(r, 0, t)$ based on this three stress distribution patterns is obtained. The specific expression of $\sigma_{z}(r, 0, t)$ refers to Table 3 .

The displacement at the center of the baseplate will be equal to the displacement of the ground, $w(r, 0, t)$ :

$$
w(t)=-\frac{p_{0} e^{i \omega t}}{G r_{0}}\left(f_{1}^{\prime}+i f_{2}^{\prime}\right),
$$

where $f_{1}^{\prime}$ and $f_{2}^{\prime}$ are the dimensionless frequencies. For each assumption of the stress distribution $f_{1}^{\prime}$ and $f_{2}^{\prime}$ refer to Table 3 .

Redefining $f_{1}=-f_{1}^{\prime}$ and $f_{2}=-f_{2}^{\prime},(6)$ can be written as

$$
w(t)=\frac{p_{0} e^{i \omega t}}{G r_{0}}\left(f_{1}+i f_{2}\right) .
$$

That is,

$$
\begin{aligned}
p_{0} e^{i \omega t} & =\frac{G r_{0}}{f_{1}+i f_{2}} w(t) \\
& =\left[-\frac{f_{1}}{f_{1}^{2}+f_{2}^{2}}+i\left(\frac{f_{2}}{f_{1}^{2}+f_{2}^{2}}\right)\right] G r_{0} w(t),
\end{aligned}
$$

where $p_{0} e^{i \omega t}$ is the driving signal, $w(t)$ is the response, and $G r_{0} /\left(f_{1}+i f_{2}\right)$ is the impedance function of the vibrational system.

Equation (7) is the dynamic model of the infinite half space where circular surface is under a uniform stress $p_{0} e^{i \omega t}$.

\subsection{Dynamic Model of Baseplate-Ground Vibrational System.} For the baseplate-ground system, if nonlinear factors are neglected, the system vibration can be treated as a superposition vibration under a vertical disturbance force $Q_{0} e^{i(\omega t+\varphi)}$ (Figure 5).

According to (8), the reaction force on the bottom of the baseplate can be written as

$$
\begin{aligned}
R & =R_{0} e^{i \omega t}=p_{0} e^{i \omega t} \\
& =\left[-\frac{f_{1}}{f_{1}^{2}+f_{2}^{2}}+i\left(\frac{f_{2}}{f_{1}^{2}+f_{2}^{2}}\right)\right] G r_{0} w(t) .
\end{aligned}
$$


TABLE 1: $F_{1}$ and $F_{2}$ for different modes of vibration.

\begin{tabular}{lccc}
\hline Mode of vibration & \multicolumn{2}{c}{ Poisson's ratio $\mu$} \\
& 0 & 0.25 & 0.5 \\
\hline Vertical vibration $\left(0<a_{0}<1.5\right)$ & $F_{1}=4.0-0.5 a_{0}^{2}$ & $F_{1}=5.3-1.0 a_{0}^{2}$ & $F_{1}=8.0-2.0 a_{0}^{2}$ \\
& $F_{2}=3.3+0.4 a_{0}$ & $F_{2}=4.4+0.8 a_{0}$ & $F_{2}=6.9$ \\
Horizontal vibration $\left(0<a_{0}<2\right)$ & $F_{1}=4.5-2.0 a_{0}^{2}$ & $F_{1}=4.8-0.2 a_{0}^{2}$ & $F_{1}=5.3-0.1 a_{0}^{2}$ \\
& $F_{2}=2.4+0.3 a_{0}$ & $F_{2}=2.5+0.3 a_{0}$ & $F_{2}=2.8+0.4 a_{0}$ \\
Swing vibration $\left(0<a_{0}<2\right)$ & $F_{1}=2.5-0.4 a_{0}^{2}$ & - & - \\
Twisting vibration $\left(0<a_{0}<1.5\right)$ & $F_{2}=0.4 a_{0}$ & $F_{1}=5.1-0.3 a_{0}^{2}$ & $F_{1}=5.1-0.3 a_{0}^{2}$ \\
& $F_{1}=5.1-0.3 a_{0}^{2}$ & $F_{2}=0.5 a_{0}$ & $F_{2}=0.5 a_{0}$ \\
\hline
\end{tabular}

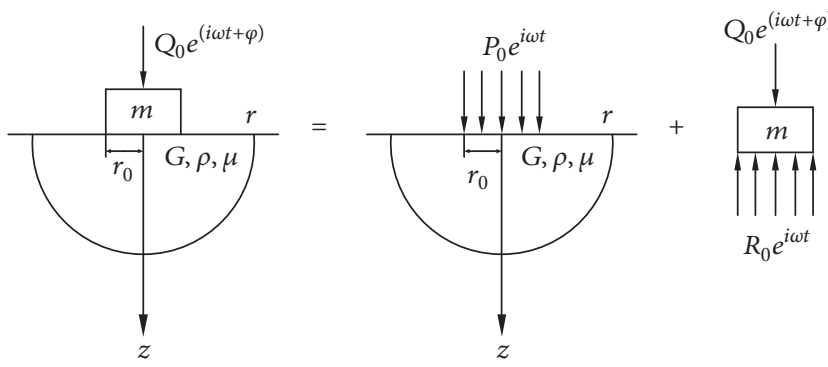

FIGURE 5: Decomposition and superposition of the baseplateground vibrational system.

Therefore, the differential equation describing the dynamics of the baseplate can be written as [19]

$$
m \frac{d^{2} w(t)}{d t^{2}}+R_{0} e^{i \omega t}=Q_{0} e^{i(\omega t+\varphi)}
$$

That is,

$$
\begin{aligned}
& m \frac{d^{2} w(t)}{d t^{2}}+\left[\frac{f_{1}}{f_{1}^{2}+f_{2}^{2}}+i\left(\frac{f_{2}}{f_{1}^{2}+f_{2}^{2}}\right)\right] G r_{0} w(t) \\
& =Q_{0} e^{i(\omega t+\varphi)}
\end{aligned}
$$

where $m$ and $w(t)$ are, respectively, the mass and the displacement response of the baseplate, $Q_{0}$ is the amplitude of the external force, and $\varphi$ is the phase difference.

For harmonic vibrations, $i \omega w(t)=\dot{w}(t)$ and $\omega=$ $\left(a_{0} / r_{0}\right) \sqrt{G / \rho}$ and (11) can be written as

$$
\begin{gathered}
m \frac{d^{2} w(t)}{d t^{2}}+\left[\frac{f_{2}}{\left(f_{1}^{2}+f_{2}^{2}\right) a_{0}} \sqrt{G \rho r_{0}^{2}}\right] w(t) \\
+\left[-\frac{f_{1}}{f_{1}^{2}+f_{2}^{2}} G r_{0}\right] w(t)=Q_{0} e^{i(\omega t+\varphi)}
\end{gathered}
$$

Under the assumptions

$$
\begin{aligned}
& F_{1}=-\frac{f_{1}}{f_{1}^{2}+f_{2}^{2}}, \\
& F_{2}=\frac{f_{2}}{\left(f_{1}^{2}+f_{2}^{2}\right) a_{0}},
\end{aligned}
$$

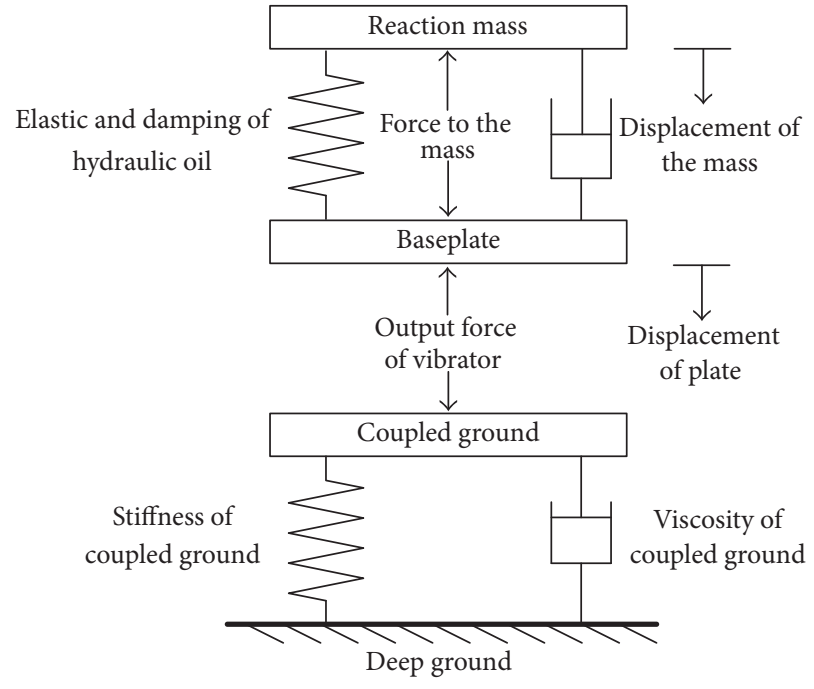

FIGURE 6: Mechanical calculation diagram for seismic vibratorground coupling system.

(12) can be simplified to

$$
\begin{aligned}
m \ddot{w} & (t)+\left[\sqrt{G \rho} r_{0}^{2} F_{2}\right] w(t)+\left[G r_{0} F_{1}\right] w(t) \\
& =Q_{0} e^{i(\omega t+\varphi)},
\end{aligned}
$$

where $\sqrt{G \rho} r_{0}^{2} F_{2}$ and $G r_{0} F_{1}$ are the stiffness $K_{z}$ and damping $C_{z}$. They are functions of the disturbance frequency $\omega$. Expressions for $F_{1}$ and $F_{2}$ are given in Table 1 for the different modes of vibration.

\section{Dynamic Model of Seismic Vibrator-Ground Coupling}

3.1. Vibrational Coupling Model. A simple seismic vibratorground coupling system is shown in Figure 6 [3]. 
The dynamic equations for the coupling system can be written as

$$
\begin{aligned}
\mathbf{M} \ddot{x}+\mathbf{C} \dot{x}+\mathbf{K} x & =Q(t), \\
\mathbf{M} & =\left[\begin{array}{cc}
m_{b} & 0 \\
0 & m_{r}
\end{array}\right], \\
\mathbf{C} & =\left[\begin{array}{cc}
C_{Z}+c_{o} & -c_{o} \\
c_{o} & c_{o}
\end{array}\right], \\
\mathbf{K} & =\left[\begin{array}{cc}
K_{Z}+k_{o} & -k_{o} \\
-k_{o} & k_{o}
\end{array}\right],
\end{aligned}
$$

where $m_{r}$ and $m_{b}$ are, respectively, the masses of the reaction mass and the baseplate, $K_{z}$ and $C_{z}$ are, respectively, the stiffness and damping of the ground, and $k_{0}$ and $c_{0}$ are, respectively, the stiffness and damping of the hydraulic oil. $Q(t)$ is the sweep signal and can be written as follows for a linear signal:

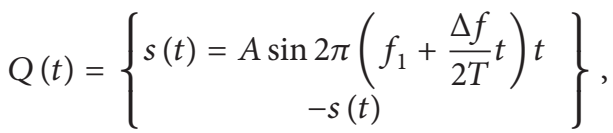

$$
\begin{aligned}
& 0<t<T,
\end{aligned}
$$

where $A$ is the amplitude, $T$ is the sweep length, $t$ is the time, $f_{1}$ and $f_{2}$ are the start frequency and end frequency, and $\Delta f=$ $f_{2}-f_{1}$ is the sweep bandwidth.

3.2. Solution of the Model. Equation (15) is solved using Newmark's integral method, with the displacement and velocity at instant $t+\Delta t(i+1)$ being expressed, respectively, as

$$
\begin{aligned}
& \mathbf{S}_{i+1}=\mathbf{S}_{i}+\dot{\mathbf{S}}_{i} \Delta t+\left[\left(\frac{1}{2}-\beta\right) \ddot{\mathbf{S}}_{i}+\beta \ddot{\mathbf{S}}_{i+1}\right] \Delta t^{2}, \\
& \dot{\mathbf{S}}_{i+1}=\dot{\mathbf{S}}_{i}+(1-\delta) \ddot{\mathbf{S}}_{i} \Delta t+\delta \ddot{\mathbf{S}}_{i+1} \Delta t
\end{aligned}
$$

where $\Delta t$ is the time interval. $\delta$ and $\beta$ are parameters related to the precision and stability, and the values are 0.5 and 0.25 , respectively.

Using (17a) and (17b), the velocity and acceleration at instant $i+1$ can be expressed approximately by the responses at instant $i$ and the displacement at instant $i+1$ :

$$
\begin{aligned}
\dot{\mathbf{S}}_{i+1}= & \frac{\delta}{\beta \Delta t}\left(\mathbf{S}_{i+1}-\mathbf{S}_{i}\right)+\left(1-\frac{\delta}{\beta}\right) \dot{\mathbf{S}}_{i} \\
& +\left(1-\frac{\delta}{2 \beta}\right) \ddot{\mathbf{S}}_{i} \Delta t, \\
\ddot{\mathbf{S}}_{i+1}= & \frac{1}{\beta \Delta t^{2}}\left(\mathbf{S}_{i+1}-\mathbf{S}_{i}\right)-\frac{1}{\beta \Delta t} \dot{\mathbf{S}}_{i}-\left(\frac{1}{2 \beta}-1\right) \ddot{\mathbf{S}}_{i} .
\end{aligned}
$$

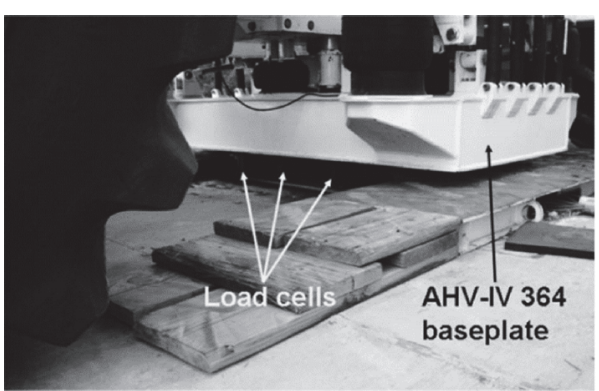

FIGURE 7: The vibrator equipped with an advanced actuator located on load cells [14].

Substitution of (18a) and (18b) into (15) yields the following equation:

$$
\begin{aligned}
(\mathbf{K} & \left.+\frac{1}{\beta \Delta t^{2}} \mathbf{M}+\frac{\delta}{\beta \Delta t} \mathbf{C}\right) \mathbf{S}_{i+1} \\
= & f_{i+1}+\mathbf{M}\left[\frac{1}{\beta \Delta t^{2}} \mathbf{S}_{i}+\frac{1}{\beta \Delta t} \dot{\mathbf{S}}_{i}+\left(\frac{1}{2 \beta}-1\right) \ddot{\mathbf{S}}_{i}\right] \\
& +\mathbf{C}\left[\frac{\delta}{\beta \Delta t} \mathbf{S}_{i}+\left(\frac{\delta}{\beta}-1\right) \dot{\mathbf{S}}_{i}+\frac{\Delta t}{2}\left(\frac{\delta}{\beta}-2\right) \ddot{\mathbf{S}}_{i}\right] .
\end{aligned}
$$

The system responses, including displacements, velocities, and acceleration, at instant $i+1$ can be obtained by substituting the solution of (19) into (18a) and (18b).

3.3. Verification of the Model. To verify the validity of the dynamic model, the force output of a real seismic vibratorground system is calculated and compared with the experiment data. The experiment was taken with an AHV-IV model-362 vibrator positioned on load cells using $5 \mathrm{~Hz}$ to $105 \mathrm{~Hz}$ in a 20-second linear sweep. A linear sweep was used to run a vibrator equipped with an advanced actuator which carries a modified hydraulic system and a new stiffer baseplate that is 2.5 times stiffer than the standard vibrator baseplate $[21,22]$. This vibrator in Figure 7 was placed on load cells from which the true vibrator-ground force can be measured. All load cells were firmly mounted on a concrete pad and the target force was set at $42000 \mathrm{lbs}$ or $186816 \mathrm{~N}$ [14]. In the experiment, it is assumed that the ground is homogeneous. Key parameters in the model and the experiment are listed in Table 2, and the parameters of the sweep signal in (16) are $A=280 \mathrm{kN}, f_{1}=5 \mathrm{~Hz}, f_{2}=105 \mathrm{~Hz}$, and $T=20 \mathrm{~s}$.

The time-history response of the output force from the model is compared with experimental results in Figure 8. Both model data and experimental results show that the vibrator output force decreases as the sweep frequency goes up. At very high frequencies, the torque motor current on the pilot servo-valve reaches its limit of $40 \mathrm{~mA}$ (milliampere). Often times, the instability of the servo-valve system occurs once the torque motor current is saturated and causes the poor vibrator performance [23]. The amplitude envelope in Figure 9 produced by model data shows a similarity to the amplitude envelope of experimental data although 
TABLE 2: Parameters of seismic vibrator-ground system.

\begin{tabular}{|c|c|c|c|c|c|c|}
\hline & Hydraulic oil & Air spring & Vibrator vehicle & Reaction mass & Baseplate & Ground \\
\hline Stiffness $(\mathrm{N} / \mathrm{m})$ & $6.25 \times 10^{5}$ & $1.6 \times 10^{5}$ & - & - & - & $1.3 \times 10^{10}$ \\
\hline Damping $(\mathrm{N} \cdot \mathrm{s} / \mathrm{m})$ & $1 \times 10^{4}$ & $1 \times 10^{6}$ & - & - & - & $7 \times 10^{6}$ \\
\hline Mass (kg) & - & - & 28,000 & 2600 & 1380 & - \\
\hline Density $\left(\mathrm{kg} / \mathrm{m}^{3}\right)$ & - & - & - & - & - & 2600 \\
\hline Area $\left(\mathrm{m}^{2}\right)$ & - & - & - & - & 2.64 & - \\
\hline Young's modulus $\left(\mathrm{N} / \mathrm{m}^{2}\right)$ & - & - & - & - & - & $2 \times 10^{6}$ \\
\hline
\end{tabular}

TABLE 3

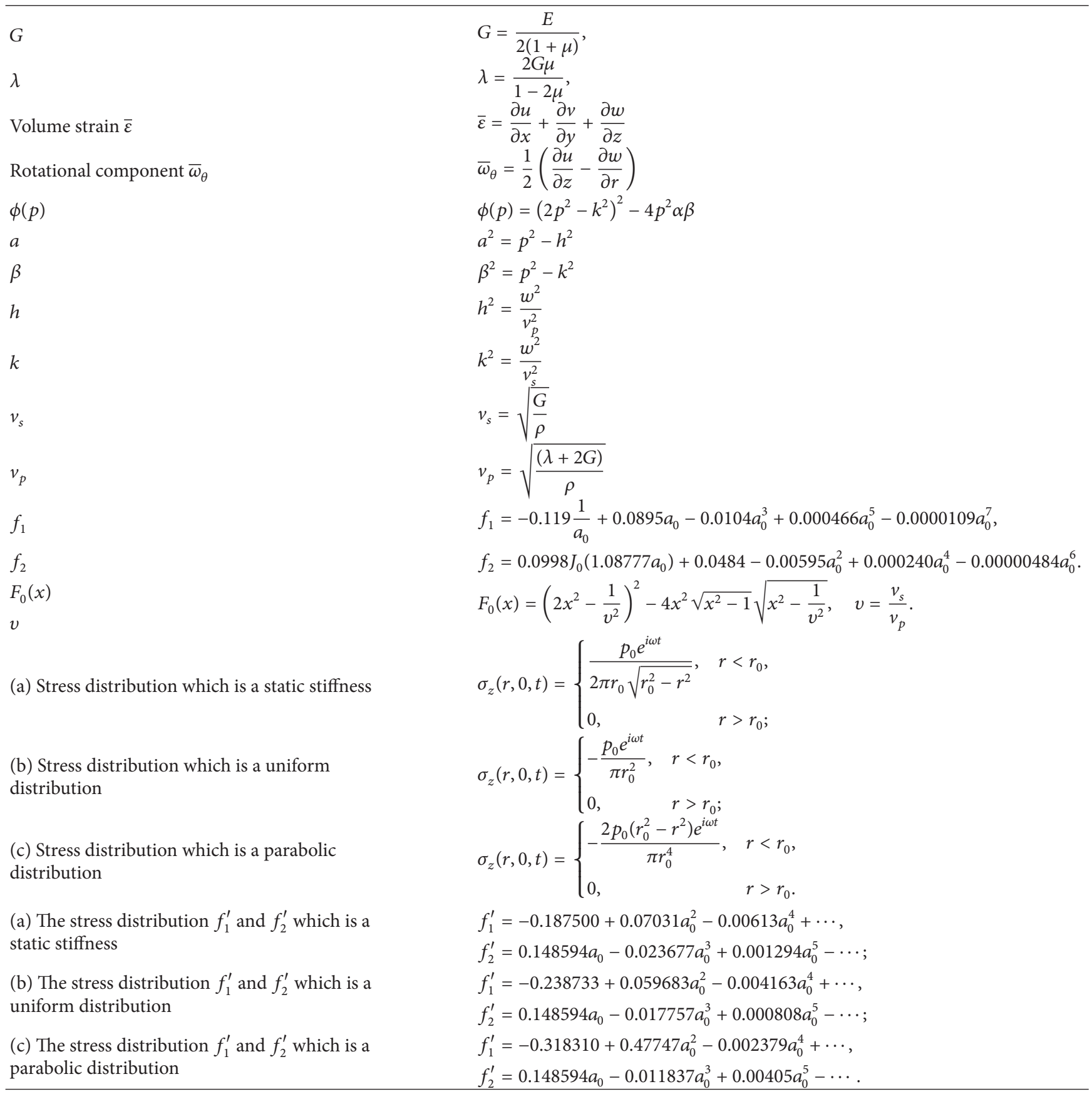




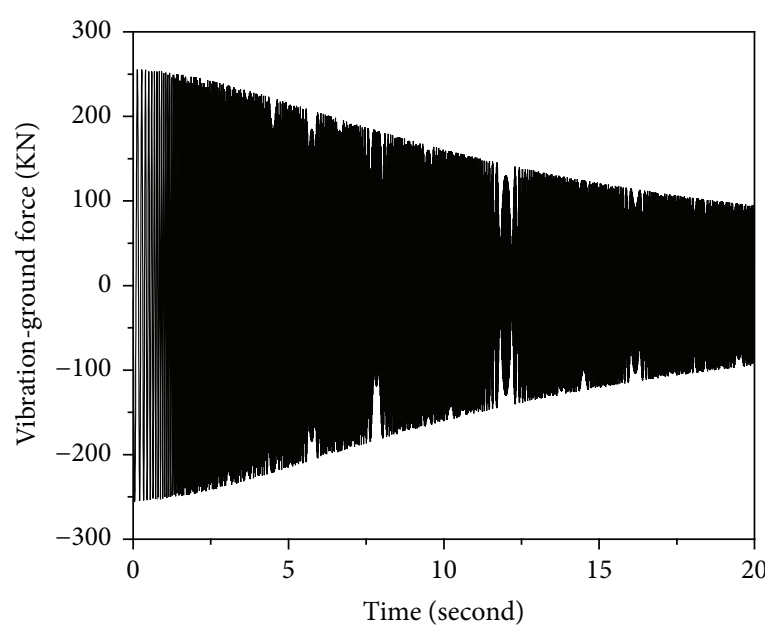

(a)

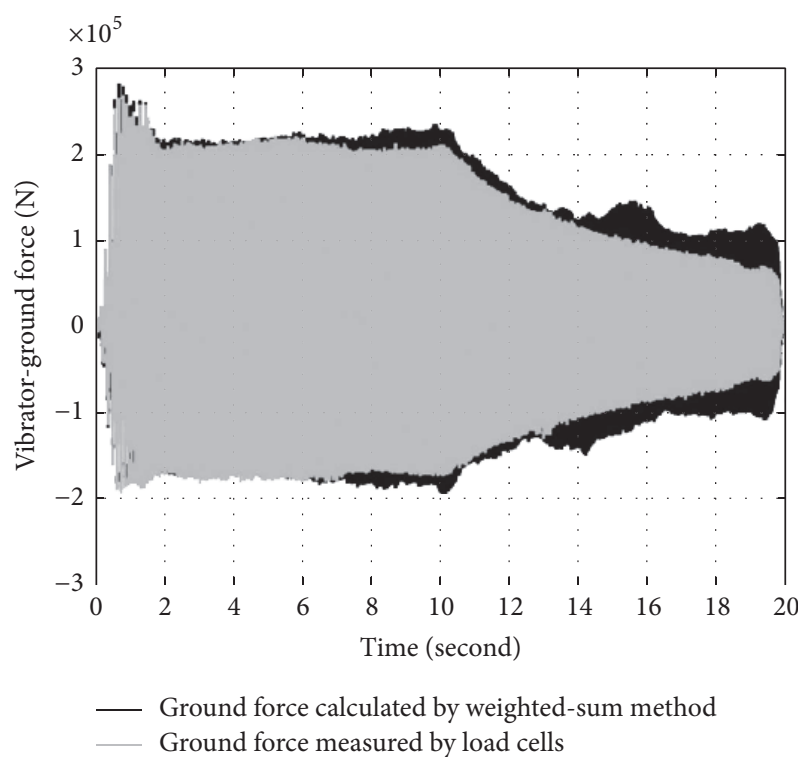

(b)

FIGURE 8: Comparison between theoretical and measured values of the output force: (a) output force time-history response from the present model; (b) output force time-history response from the weighted-sum method and experimental results [14].

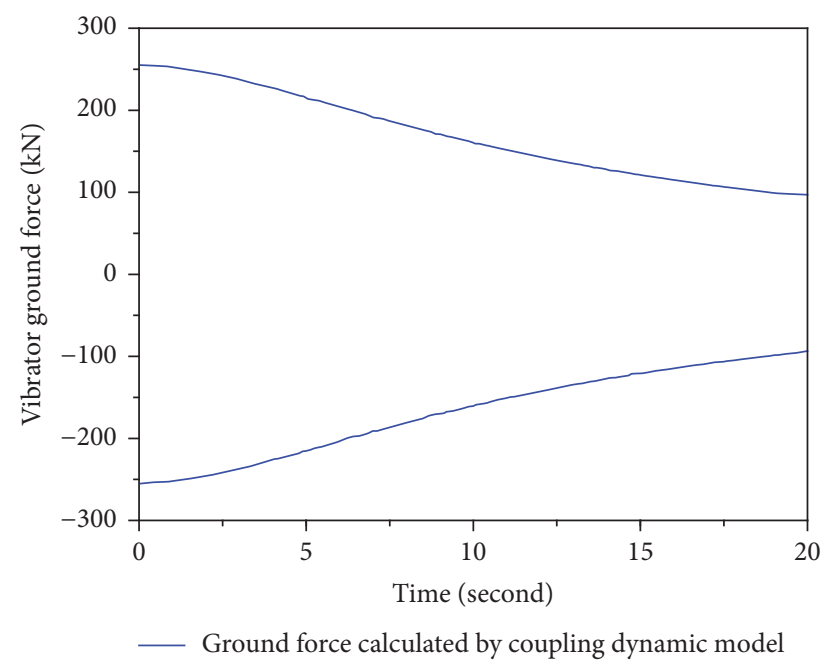

Figure 9: Comparison of output force amplitude time-history responses. some discrepancy is still seen. This shows that the model is reasonable and can be used to estimate the impact of parameters in vibrator-ground system on the vibrator output force.

\section{Dynamic Characteristics Analysis}

4.1. Modal Analysis. The characteristic equation for the dynamic equations (15) of the coupling system can be written as

$$
\mathbf{B}=\left|\mathbf{K}-\omega^{2} \mathbf{M}\right|=\left|\begin{array}{cc}
K_{Z}+k_{o}-\omega^{2} m_{b} & -k_{o} \\
-k_{o} & k_{o}-\omega^{2} m_{r}
\end{array}\right|=0 .
$$

The natural frequency of the system can be obtained by solving this characteristic equation and is found to be

$$
\omega^{2}=\frac{m_{r}\left(K_{z}+k_{o}\right)+m_{b} k_{o} \pm \sqrt{\left[m_{r}\left(K_{Z}+k_{o}\right)+m_{b} k_{o}\right]^{2}-4 m_{b} m_{r}\left[\left(K_{z}+k_{o}\right) k_{o}-k_{o}^{2}\right]}}{2 m_{b} m_{r}} .
$$

The vibrational modal matrix of the coupling system can be written as

$$
\mathbf{P}=\left[\begin{array}{cc}
1 & 1 \\
\frac{K_{Z}+k_{o}-\omega_{1}^{2} m_{b}}{-k_{o}} & \frac{K_{Z}+k_{o}-\omega_{2}^{2} m_{b}}{-k_{o}}
\end{array}\right] .
$$

If a first-order vibration system is used to describe the vibrator actuator motion at low frequencies, the reaction mass and baseplate motions can be depicted as a synchronous vibration where the natural frequency is at $2.77 \mathrm{~Hz}$ (Figure 10(a)). The displacement amplitude of the reaction mass is much greater than the baseplate. This means that 


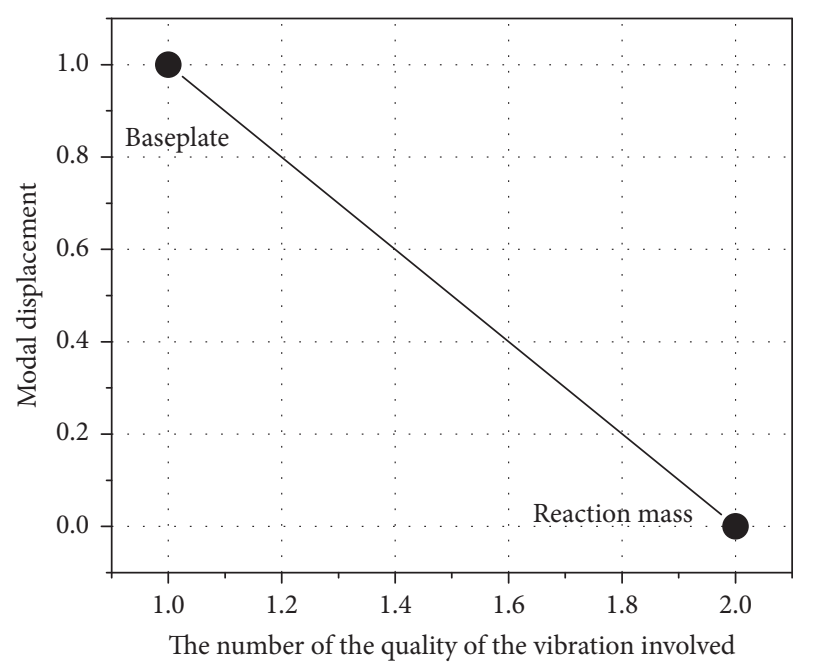

(a)

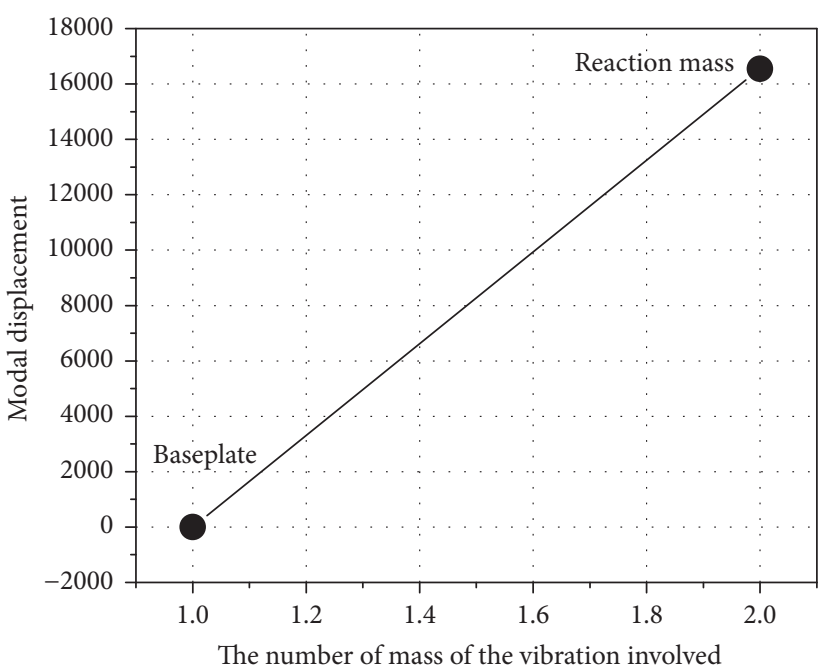

(b)

FIGURE 10: First two vibrational modes of the coupled system: (a) first-order mode and (b) second-order mode.

contribution to the vibrator output force from the baseplate motion can be neglected and decoupling hardly occurs.

As the frequency goes up, a second-order vibration system is capable of describing the reaction mass and baseplate motions where the natural frequency is at $488.7 \mathrm{~Hz}$ (Figure 10(b)). The displacement amplitude of the baseplate becomes greater than the reaction mass. As the vibration frequency approaches the natural frequency, the phase difference of the reaction mass and baseplate becomes large and goes to 180 degrees apart. This out-phase relationship will possibly cause the decoupling. It is important that the sweep frequency should not be close to this natural frequency.

4.2. Effects of System Parameters on Vibrational Output. The effects of reaction (inertial) mass and baseplate mass on the vibrational output are shown in Figures 11 and 12 which illustrate the effects of the masses of the reaction mass and baseplate on the vibrator output force, respectively. It can be seen from Figures 11 and 12 that an increase of the reaction mass results in an increase of the vibrator force amplitude while an increase of the mass of the baseplate results in a decrease of the vibrator force amplitude. These findings provide guidelines for the design of the vibrator actuator. It is authors' opinion that the mass ratio of the reaction mass and the baseplate should be kept at $3: 1$.

Figure 13 displays how the elastic modulus of the ground affects the vibrator output force. It can be observed that increasing the elastic modulus of the ground will improve the vibrator output force. Increasing the elastic modulus means that the ground becomes stiff. Although it helps improve the amplitude of the vibrator output force, it reduces the force-energy that is transmitted to the deep ground. For example, a vibrator vibrating on a piece of limestone will produce an improved force amplitude. However, the forceenergy radiated into deep ground will become poor.

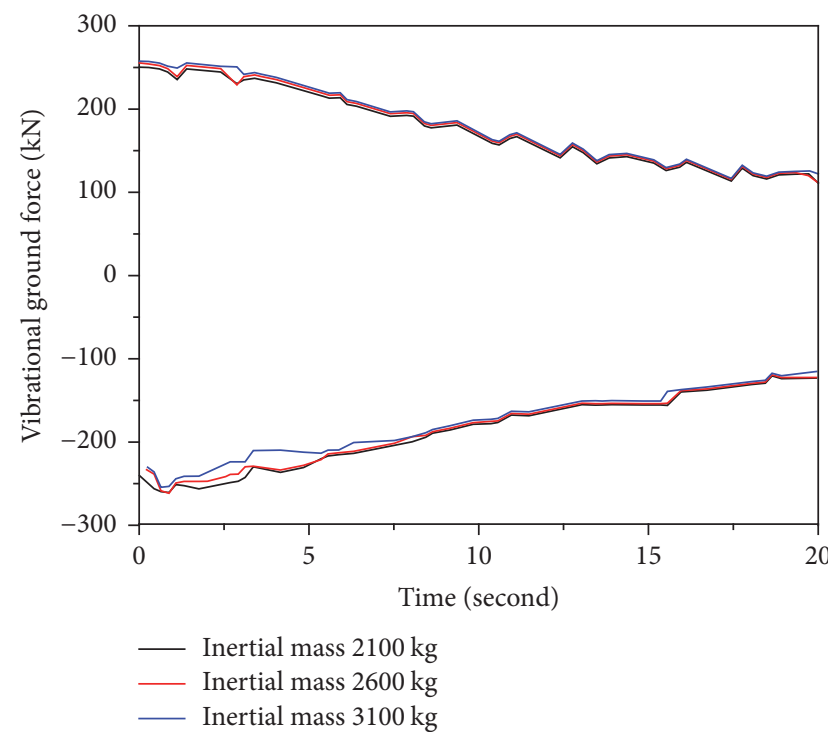

FIGURE 11: Effect of reaction (inertial) mass on vibrational output.

4.3. Effects of System Parameters on Phase Difference. To obtain a vibrational output force of high quality, it is necessary that the phase difference between the sweep signal and the vibrational output be as small as possible. To investigate the influence of the system parameters on the phase difference, the following harmonic signal is used to replace the actual linear sweep signal shown in (16):

$$
s(t)=280 \sin 2 \pi f t .
$$

Figure 14 shows the vibrational output response under a harmonic sweep signal. It can be seen that, with increasing frequency of the sweep signal, the phase difference becomes larger. 


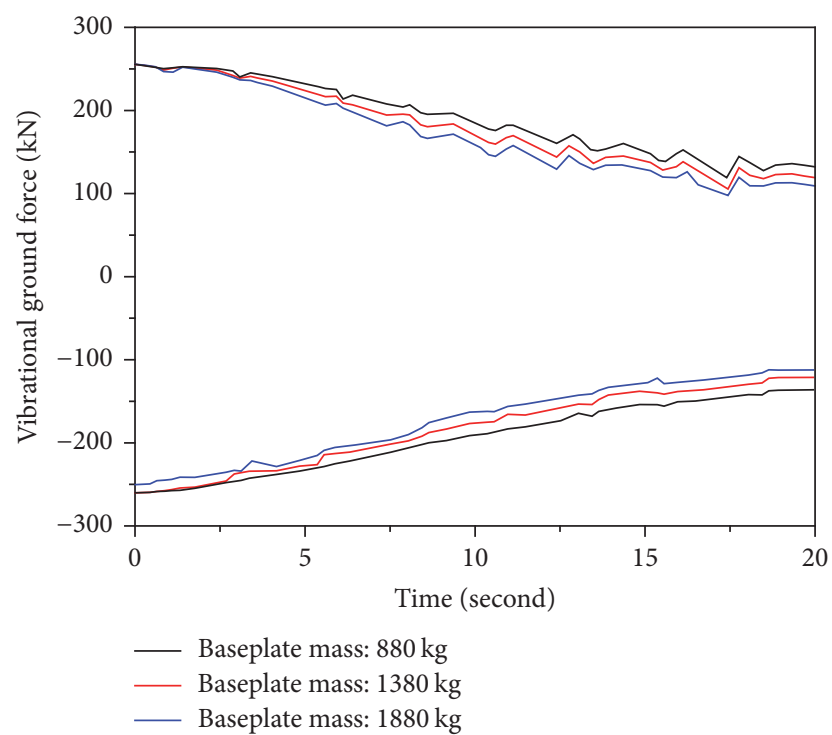

FIGURE 12: Effect of baseplate mass on vibrational output.

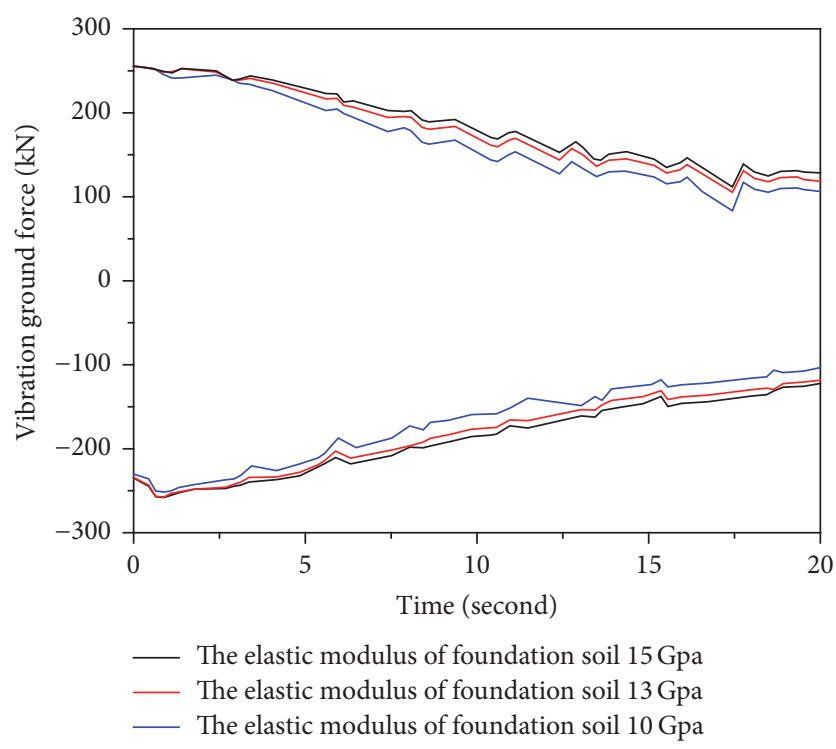

Figure 13: Effect of elastic modulus on vibrational output.

To further investigate the influence of the frequency of the sweep signal on the phase difference, the load vector of the system dynamic (15) is replaced by the following expression:

$$
\mathrm{Q}(t)=\left\{\begin{array}{c}
s(t)=280 \sin \omega t \\
-s(t)
\end{array}\right\}, \quad 0 \leq t \leq T .
$$

The steady-state solution of (15) can be assumed to be

$$
\begin{aligned}
& X_{1}=A_{1} \sin \omega t+A_{2} \cos \omega t, \\
& X_{2}=B_{1} \sin \omega t+B_{2} \cos \omega t,
\end{aligned}
$$

where $X_{1}$ and $X_{2}$ are the displacement state solutions for the vibrating plate and hammer and $A_{1}, A_{2}, B_{1}$, and $B_{2}$ are undetermined coefficients.
Substituting $X_{1}, \dot{X}_{1}$, and $\ddot{X}_{1}$ and $X_{2}, \dot{X}_{2}$, and $\ddot{X}_{2}$ into (15) and separating the sine and cosine functions yield the following equations:

$$
\begin{aligned}
& -\left(m_{r} \omega^{2}+k_{0}\right) A_{1}+k_{0} B_{1}+c_{0} \omega A_{2}-c_{0} \omega B_{1}=-F_{m} \\
& -c_{0} \omega A_{1}+c_{0} \omega B_{1}-\left(m_{r} \omega^{2}+k_{0}\right) A_{2}+k_{0} B_{2}=0 \\
& k_{0} A_{1}+\left(k_{z}-k_{0}-m_{b} \omega^{2}\right) B_{1}-c_{0} \omega A_{2} \\
& \quad+\left(c_{0}-c_{z}\right) \omega B_{2}=F_{m} \\
& c_{0} \omega A_{1}+\left(c_{z}-c_{0}\right) \omega B_{1}+k_{0} A_{2} \\
& \quad+\left(k_{z}-k_{0}-m_{b} \omega^{2}\right) B_{2}=0 .
\end{aligned}
$$

The undetermined coefficients $A_{1}, A_{2}, B_{1}$, and $B_{2}$, and thus $X_{1}$ and $X_{2}$, can be determined by solving (26a), (26b), (26c), and (26d).

The force transmitted to the ground through the baseplate can be written as

$$
F_{D}=k_{z} X_{1}+c_{z} \dot{X}_{1}
$$

Substituting $X_{1}$ into this equation then gives

$$
\begin{aligned}
F_{D} & =F_{A} \cos (\omega t+\alpha), \\
F_{A} & =\sqrt{\left(k_{z} A_{2}+c_{z} A_{1} \omega\right)^{2}+\left(k_{z} A_{1}-c_{z} A_{2} \omega\right)^{2}}, \\
\alpha & =\arctan \left(\frac{k_{z} A_{1}-c_{z} A_{2} \omega}{k_{z} A_{2}+c_{z} A_{1} \omega}\right),
\end{aligned}
$$

where $F_{A}$ and $\alpha$ are, respectively, the amplitude of the vibrational output and the phase difference between the sweep signal and the vibrational output.

Figure 15 shows that the effect of the reaction mass on the phase difference is significant, with greater reaction mass leading to a bigger phase difference, but, as shown in Figure 16, there is no obvious effect of baseplate mass on the phase difference.

Figure 17 shows that there is an obvious influence of baseplate area on phase difference, with greater baseplate area leading to a smaller phase difference. It can be seen from Figure 18 that the greater the ground stiffness, the greater the phase difference.

\section{Conclusions}

The dynamic characteristics of a seismic vibrator-ground coupling system have been investigated by analyzing the system's vibrational model and the influence of the main system parameters on the vibrational output and the phase difference between the input sweep signal and the vibrational output. The following conclusions can be drawn from the results obtained:

(1) The main vibrational modes of the coupling system are the first- and second-order modes with frequencies $2.77 \mathrm{~Hz}$ and $488.7 \mathrm{~Hz}$, the latter being reverse 


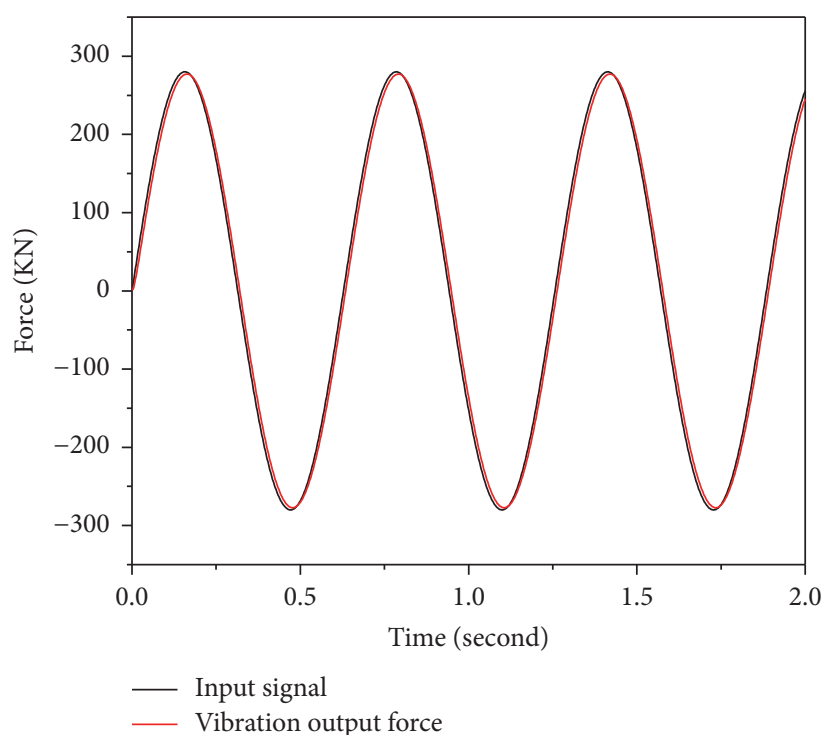

(a)

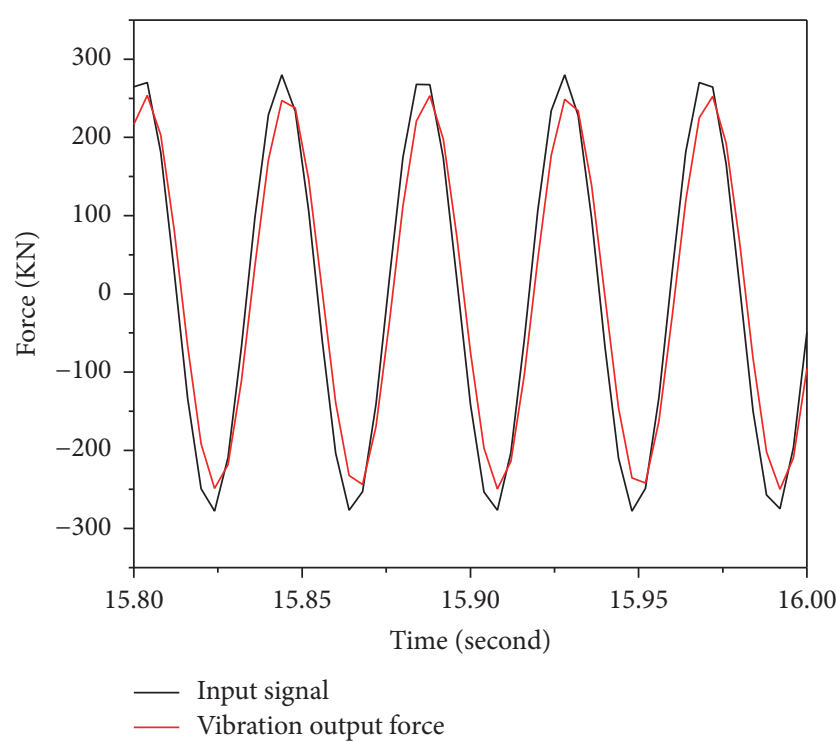

(b)

FigURE 14: Phase comparison between input signal and vibrational output: (a) sweep signal frequency $f=1.59 \mathrm{~Hz}$ and (b) sweep signal frequency $f=23.89 \mathrm{~Hz}$.

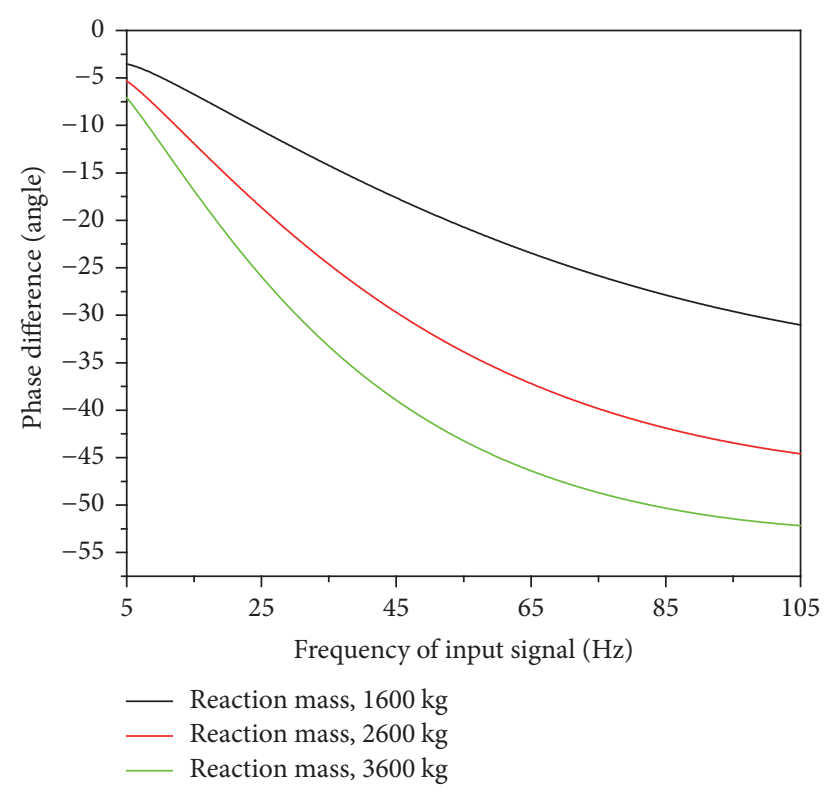

FIGURE 15: Effect of reaction mass on phase difference for a baseplate mass of $2600 \mathrm{~kg}$.

movement of the reaction mass and baseplate will lead to an output phase difference of 180 degrees. This outphase relationship will possibly cause the decoupling. Therefore, in an actual vibrator design, the frequency of the sweep signal should be lower than the secondorder frequency of the system.

(2) The design of the two most important components of the coupling system, namely, the baseplate and the

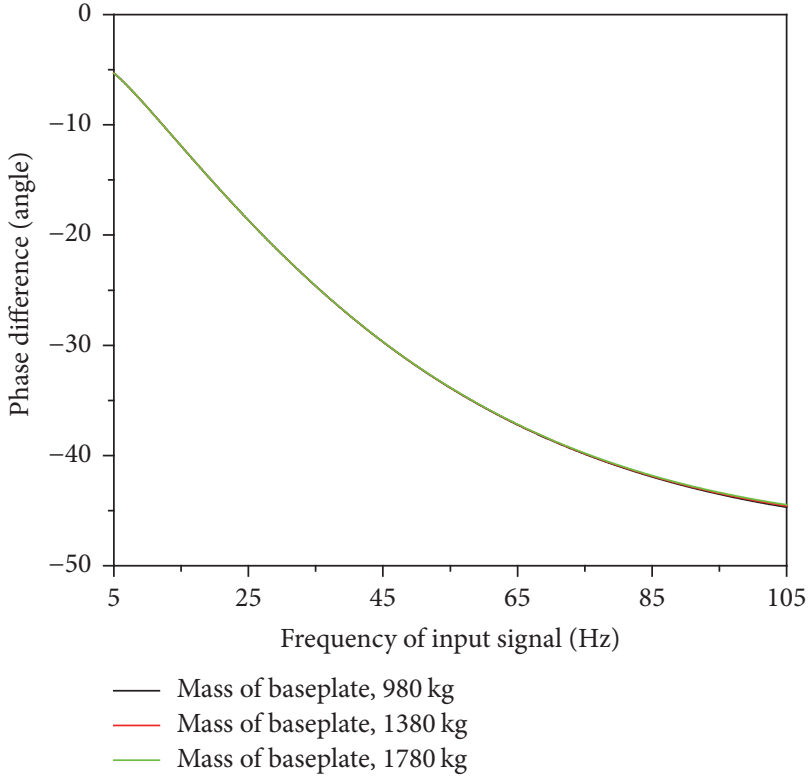

FIGURE 16: Effect of baseplate mass on phase difference for a reaction mass of $1380 \mathrm{~kg}$.

reaction mass, should aim at improving the amplitude of the vibrational output and reducing the phase difference. It is found in this paper that an increase of the reaction mass results in an increase of the vibrator force amplitude while an increase of the mass of the baseplate results in a decrease of the vibrator force amplitude. These findings provide guidelines for the design of the vibrator actuator. The mass ratio of the reaction mass and the baseplate should be kept at $3: 1$. 


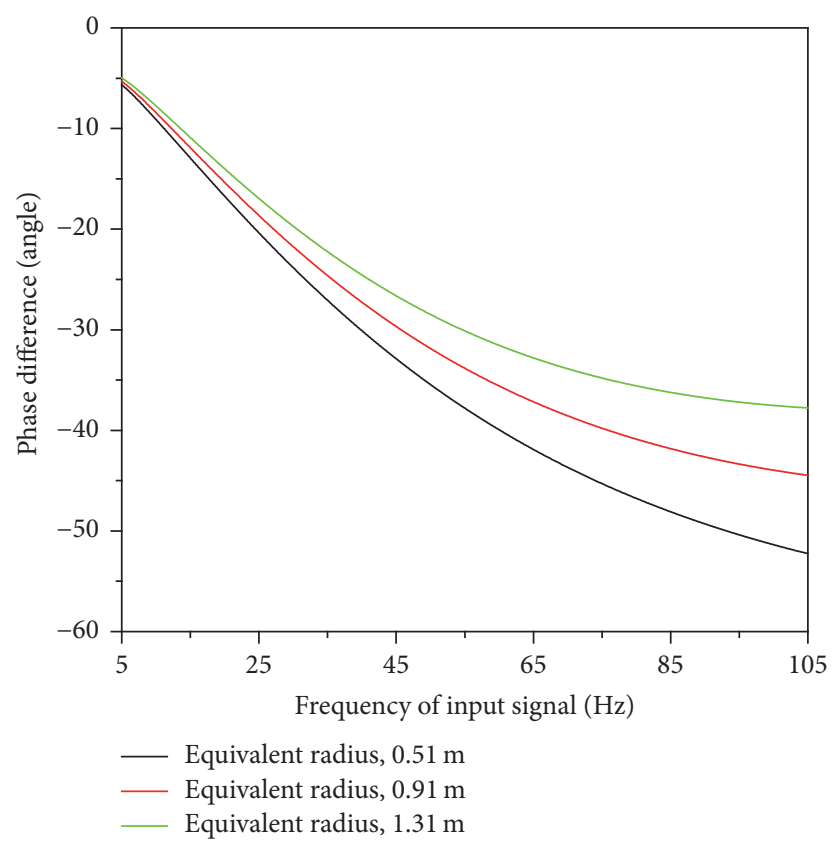

FIGURE 17: Effect of baseplate area on phase difference for a baseplate mass of $1380 \mathrm{~kg}$ and a reaction mass of $2600 \mathrm{~kg}$.

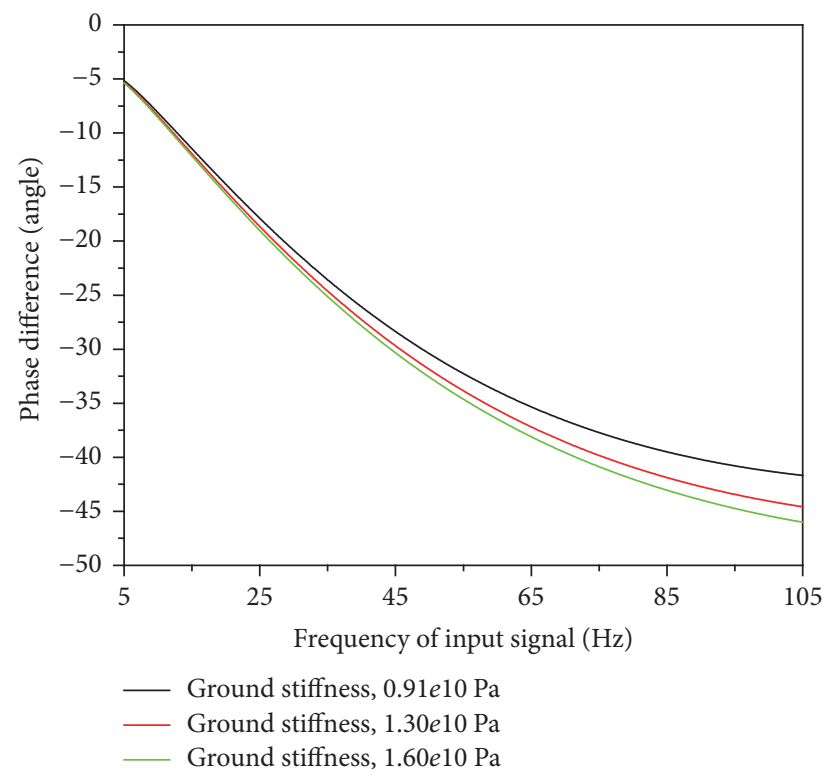

FIgURE 18: Effect of ground stiffness on phase difference for a baseplate mass of $1380 \mathrm{~kg}$ and a reaction mass of $2600 \mathrm{~kg}$.

(3) The physical properties of the ground will affect the natural frequency of the coupling system and ultimately the quality of the output signal. Therefore, the sweep signal should be determined according to the physical properties of the ground and the modal analysis of the coupled system. That is, the frequency of the sweeping signal should be kept away from the frequency of second-order vibration of the vibratorground system.

\section{Conflicts of Interest}

The authors declare that there are no conflicts of interest regarding the publication of this paper.

\section{References}

[1] Z. Q. Huang, Y. P. Ding, Z. F. Tao, L. Hao, and G. Li, "Research status and development direction of vibroseis vibrator baseplate at home and abroad," Chinese Journal of Oil Field Equipment, vol. 44, no. 6, pp. 1-5, 2015.

[2] W. L. Chapman, G. L. Brown, and D. W. Fair, "The Vibroseis system: a high-frequency tool," Journal of Geophysical, vol. 46, no. 12, pp. 1657-1666, 1981.

[3] J. J. Sallas and R. M. Weber, "The amplitude and phase response of a seismic vibrator' by W. E. Lerwill," Geophysical Prospecting, vol. 30, no. 6, pp. 935-938, 1982.

[4] K. P. Allen, M. L. Johnson, and J. S. May, "High fidelity vibratory seismic (HFVS) method for acquiring seismic data," in Proceedings of the SEG Technical Program Expanded Abstracts 1998, pp. 140-143.

[5] A. V. Lebedev and I. A. Beresnev, "Nonlinear distortion of signals radiated by vibroseis sources," Journal of Geophysical, vol. 69, no. 4, pp. 968-977, 2004.

[6] N. Nagarajappa and D. Wilkinson, "Source measurement effect on high fidelity vibratory seismic separation," Geophysical Prospecting, vol. 58, no. 1, pp. 55-68, 2010.

[7] C. Saragiotis, P. Scholtz, and C. Bagaini, "On the accuracy of the ground force estimated in vibroseis acquisition," Geophysical Prospecting, vol. 58, no. 1, pp. 69-80, 2010.

[8] R. Zhang, Y. M. Chen, and S. L. Xie, "Analysis of dynamic characteristics of micro-vibroseis," Equipment for Geophysical Prospecting, vol. 24, no. 2, pp. 91-95, 2014 (Chinese).

[9] Z. Wei, "Pushing the vibrator envelope: extending low and high frequency limits," First Break, vol. 26, no. 3, pp. 37-43, 2008.

[10] Z. Wei, "Pushing the vibrator ground-force envelope towards low frequencies," Geophysical Prospecting, vol. 57, no. 1, pp. 151$161,2009$.

[11] Z. Wei, "Modelling and modal analysis of seismic vibrator baseplate," Geophysical Prospecting, vol. 58, no. 1, pp. 19-32, 2010.

[12] G. M. Zheng, X. M. Lei, H. Zhang, and Z. F. Chen, "Researches and simulation of vibroseis signal generator based on MATLAB GUI," Geophysical and Geochemical Exploration, vol. 37, no. 1, pp. 160-164, 2013 (Chinese).

[13] W. Sun, "Vibration output simulation of controlled source," Chinese Journal of Geophysical Prospecting Equipment, vol. 22, no. 2, pp. 79-82, 2012.

[14] Z. Wei, T. F. Phillips, and M. A. Hall, "Fundamental discussions on seismic vibrators," Journal of Geophysical, vol. 75, no. 6, pp. W13-W25, 2010.

[15] M. Hall, "Analysis of field tests with an improved hydraulic vibrator," SEG Technical Program Expanded Abstracts, vol. 28, no. 1, pp. 104-108, 2009.

[16] W. T. van Horssen and N. V. Gaiko, "On the transverse, low frequency vibrations of a traveling string with boundary damping," Journal of Vibration and Acoustics, Transactions of the ASME, vol. 137, no. 4, Article ID 041004, 2015.

[17] J. M. Tian and C. Z. Liang, "Foundation design of dynamic machine foundation on natural base," Cement Technology, vol. 6, pp. 85-89, 2014 (Chinese). 
[18] R. J. Yan, Y. S. Wang, and Q. Y. Han, Introduction to Semi Space Theory of Dynamic Foundation, China Building Industry Press, Beijing, China, 1981.

[19] H. J. Qian, D. M. Zhang, and J. X. Wang, Dynamic Machine Foundation Design, China Building Industry Press, Beijing, China, 1980.

[20] B. D. Wu and L. B. Wu, "Analog method for the equivalent lumped system of foundation half-space theories and associated field measurements," China Earthquake Engineering Journal, vol. 37, no. 4, pp. 1029-1036, 2015 (Chinese).

[21] Z. Wei, "Reducing harmonic distortion on vibrators-stiffening the vibrator baseplate," in Proceedings of the 70th EAGE Conference and Exhibition incorporating SPE EUROPEC 2008, Rome, Italy, June 2008.

[22] Z. Wei, "How good is the weighted-sum estimate of the vibrator ground force?" The Leading Edge, vol. 28, no. 8, pp. 960-965, 2009.

[23] Z. Wei, "Pushing the vibrator envelope-how high can we go?" in Proceedings of the 69th EAGE Conference and Exhibition incorporating SPE EUROPEC '07, London, UK, June 2007. 


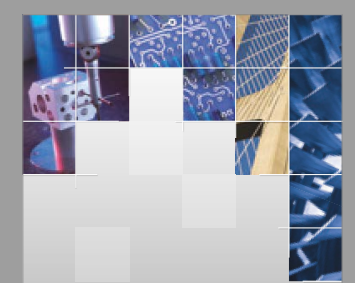

\section{Enfincering}
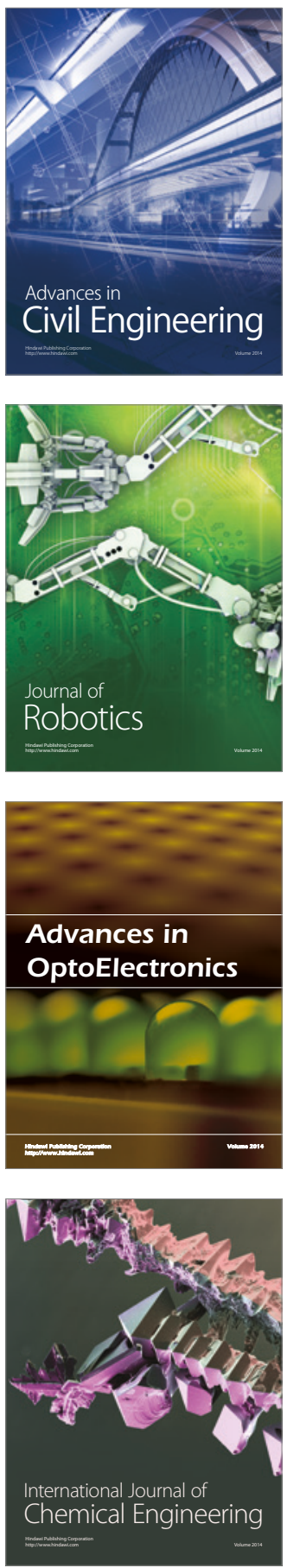

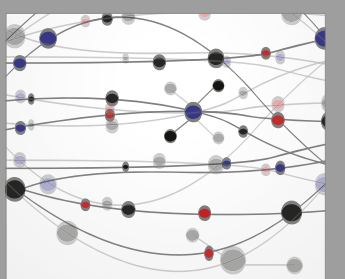

The Scientific World Journal

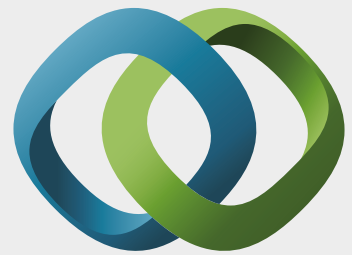

\section{Hindawi}

Submit your manuscripts at

https://www.hindawi.com
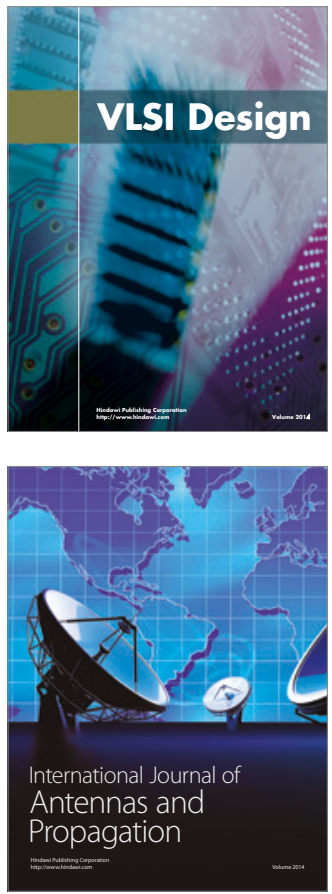

\section{Rotating}

Machinery
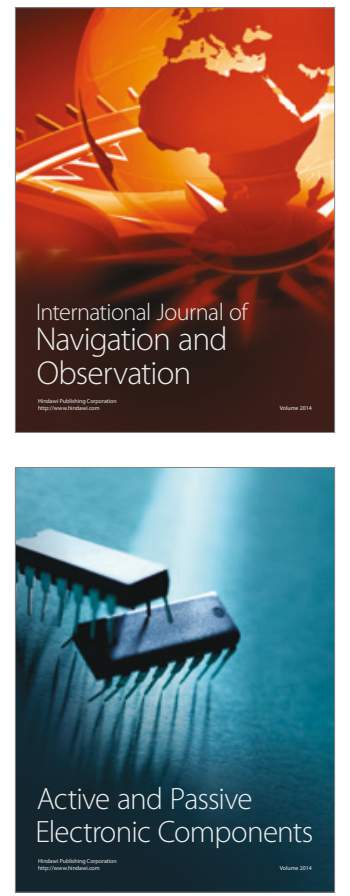
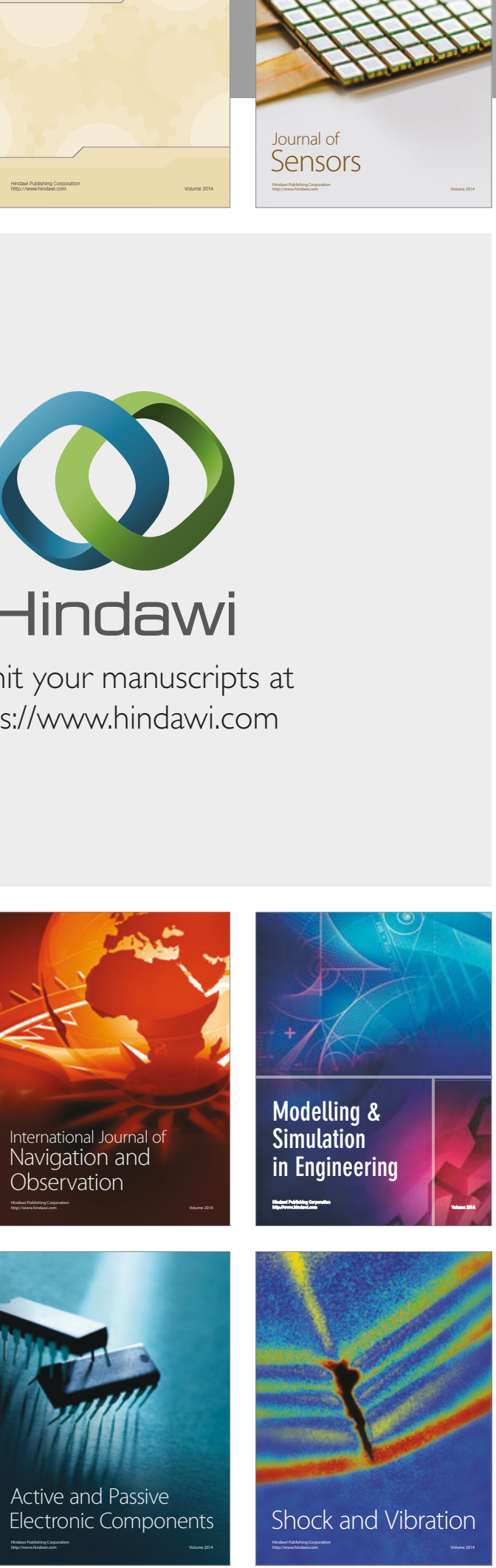
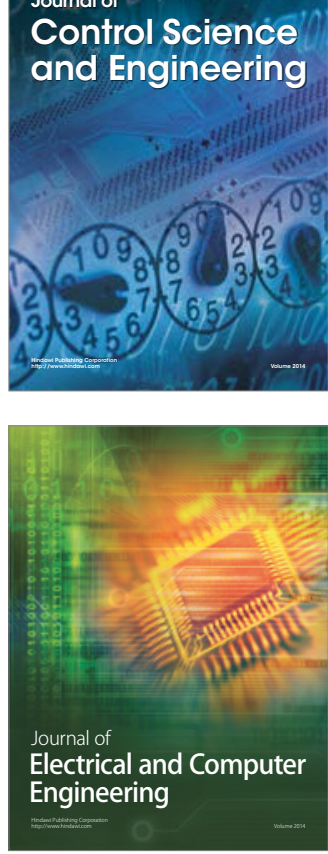

Distributed

Journal of

Control Science

and Engineering
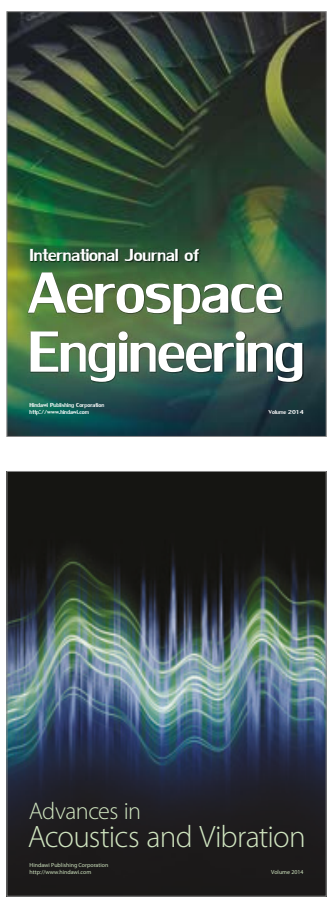

Sensor Networks 